\title{
Ambiguous value of Haemophilus influenzae isolation in Guillain-Barré and Fisher syndromes
}

\author{
M Koga, S Koike, K Hirata, N Yuki
}

J Neurol Neurosurg Psychiatry 2005;76:1736-1738. doi: 10.1136/jnnp.2005.065359

Background: Haemophilus influenzae is considered a causative agent of Guillain-Barré syndrome (GBS) and Fisher syndrome, but the frequency of this infection in $G B S$ is controversial.

Objective: To determine whether isolation of $H$ influenzae indicates it is a causative agent in GBS and Fisher syndrome. Results: Four (15\%) of 27 patients with GBS and Fisher syndrome in whom $\mathrm{H}$ influenzae was isolated were also seropositive for Campylobacter jejuni. Antiganglioside lgG antibodies in these four patients did not cross react with their $H$ influenzae lipo-oligosaccharides, whereas antiganglioside antibodies in the four patients with positive serology for $H$ influenzae did.

Conclusions: The findings suggest that $H$ influenzae isolation is not always indicative of the causative agent in these syndromes and that tests for other infections should be made, even in cases of positive culture.

G uillain-Barré syndrome (GBS) is a heterogeneous disorder in which various infections may occur before and after neurological onset. Such preceding infections as Campylobacter jejuni, cytomegalovirus, Epstein-Barr virus, and Mycoplasma pneumoniae are generally accepted as causes of GBS. ${ }^{1}$ The antecedent infectious agent is usually determined by a serological test or isolation of the agent after GBS onset, sometimes making it difficult to distinguish the antecedent infection from complications of GBS itself. This is a problem, particularly in the isolation of respiratory infectious agents, because nosocomial respiratory tract infections resulting from aspiration pneumonia and intratracheal intubation are common in GBS. ${ }^{2}$

Haemophilus influenzae, a major pathogen of community acquired respiratory infection, is considered a causative agent of GBS and Fisher syndrome, but the frequency of this infection in GBS is controversial. ${ }^{13-6}$ It is hypothesised that ganglioside epitopes on $H$ influenzae lipo-oligosaccharide (LOS) induce autoantibodies against GMl and GQ1b, thereby resulting in neuropathy. ${ }^{36}$ Identification of antecedent $H$ influenzae infection is important in clinical terms because it is an indicator of rapid recovery from GBS. ${ }^{7} \mathrm{H}$ influenzae isolation is the standard diagnostic procedure for this infection, but it is not clear whether isolation of the organism in cases of GBS or Fisher syndrome necessarily means that it is the cause of those disorders. We investigated other possible antecedent infections in patients with $H$ influenzae isolations and examined whether the isolation indicates that it is a causative agent in GBS and Fisher syndrome.

\section{METHODS}

We examined recent infections (H influenzae, $C$ jejuni, cytomegalovirus, and $M$ pneumoniae) serologically, as described elsewhere, $^{6}$ in 27 patients with GBS $(n=11)$, Fisher syndrome $(\mathrm{n}=14)$, and overlapping Fisher syndrome/GBS $(\mathrm{n}=2)$, from whom $H$ influenzae had been isolated in sputum or swab cultures. All the strains were isolated shortly after neurological onset, and artificial ventilation was needed for five patients after culture sampling. Serotypes of the isolates were all non-typable, the biotypes predominantly II and III, like the isolates of uncomplicated respiratory infections. There was a history of antecedent respiratory infection symptoms in 21 patients $(78 \%)$.

Serum IgG antibodies to GM1, GMlb, GM2, GDla, GalNAc-GDla, GDlb, GTla, GTlb, and GQlb were assayed by enzyme linked immunosorbent assay (ELISA), as reported elsewhere. ${ }^{8}$ An absorption study then was done to examine whether these antiganglioside antibodies cross reacted with the LOS on the isolates. Crude LOS was prepared as described previously. ${ }^{6}$ A $100 \mu \mathrm{l}$ portion of diluted serum, which gave an optical density of between 1.0 and 2.0 for antiganglioside antibodies, was incubated in LOS precoated wells (LOS lysate $1 \mu \mathrm{l}$ per well) of microtitre plates at $4^{\circ} \mathrm{C}$ for 48 hours. The supernatant was used as the primary antibody in the standard ELISA. Absorption rates are expressed as percentages of the optical densities obtained with and without absorption treatment.

\section{RESULTS}

Antiganglioside IgG antibodies were detected in 10 of 11 GBS patients (91\%) and in all 16 Fisher syndrome patients. Frequent antibodies were anti-GMl (73\%) and anti-GMlb $(55 \%)$ in the GBS patients, and anti-GQIb and anti-GTla (both 100\%) in the Fisher syndrome patients.

Four patients (15\%: three GBS; one Fisher syndrome) had positive serology for $C$ jejuni and seven (26\%: five Fisher syndrome; one GBS; one Fisher syndrome/GBS) had positive serology for $H$ influenzae, but none for both. All the patients were negative for cytomegalovirus and $M$ pneumoniae. Of the four $C$ jejuni seropositive patients, one had a previous history of soft faeces, but the others had no preceding gastrointestinal symptoms (table 1). Follow up serum was available for one $C$ jejuni seropositive patient (patient 3 , table 1 ) who had a significant anti-C jejuni IgA titre decrease during the recovery phase of illness. IgG antibodies to GMl, GMlb, GTla, or GQIb were detected in all four patients. In contrast, all seven patients with positive serology for $H$ influenzae infection had a previous history of respiratory infection symptoms. AntiGQlb and anti-GTla IgG antibodies were detected in six of these and anti-GMl IgG antibody in the seventh.

Cross reactivity of antiganglioside IgG antibodies to the LOSs of their $H$ influenzae isolates was examined in eight patients, four with positive serology for $C$ jejuni and four for $H$ influenzae. Cross reactivity was absent in the four who had positive $C$ jejuni serology, whereas it clearly was present in at

Abbreviations: GBS, Guillain-Barré syndrome; LOS, lipooligosaccharide 
Table 1 Patients from whom $H$ influenzae was isolated and who had positive serology for recent $C$ jejuni infection

\begin{tabular}{llllll}
\hline Patient & Age (years)/sex & Diagnosis & Antecedent symptoms & Artificial ventilation & lgG antibody against \\
\hline 1 & $5 / F$ & GBS & RTI and headache & Yes & GMI and GTla \\
2 & $33 / F$ & GBS & Soft faeces & No & GM1b \\
3 & $67 / F$ & GBS & Fever and general fatigue & No & GMI \\
4 & $66 / M$ & RS & RTI & GM1b, GTla, and GQ1b \\
\hline FS, Fisher syndrome; GBS, Guillain-Barré syndrome; RTI, respiratory tract infection. & & \\
\hline
\end{tabular}

least three of the four $H$ influenzae seropositive patients examined (fig 1). The target ganglioside of the antibodies was not related to cross reactivity (data not shown).

\section{DISCUSSION}

We found that $15 \%$ of our patients from whom $H$ influenzae was isolated had positive serology for recent $C$ jejuni infection. The specificity of the $C$ jejuni serological assay system used is $97 \%^{6}$; moreover, a significant titre decrease during the recovery phase was confirmed in one patient, indicating that there were no false positive results. In GBS and Fisher syndrome after $H$ influenzae and $C$ jejuni infections it is hypothesised that the ganglioside mimics present on the bacterial LOSs produce antiganglioside antibody. ${ }^{9}$ The antibodies in our $H$ influenzae seropositive patients did cross react with their isolate LOSs, whereas those in the $C$ jejuni seropositive patients did not. This suggests that production of antiganglioside antibodies in the latter patients was not triggered by $H$ influenzae, although $H$ influenzae infection had certainly occurred, either before or after the neurological onset. It also is noteworthy that only one of four $C$ jejuni seropositive patients had a history of preceding gastrointestinal symptoms, but this does not necessarily indicate false positive results in our $C$ jejuni serology assay because $C$ jejuni

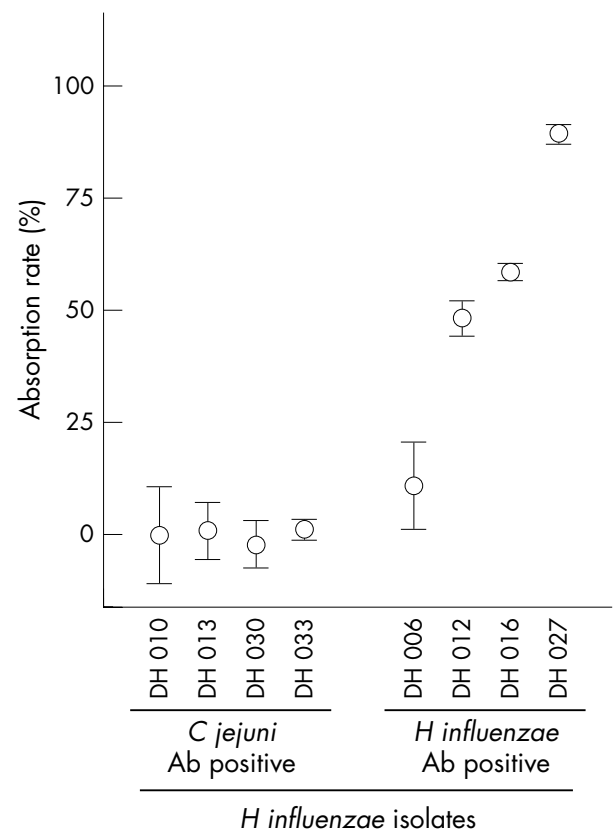

Figure 1 Cross reactivity of antiganglioside $\lg G$ antibodies in $H$ influenzae-isolate patients to their isolate lipo-oligosaccharide (LOS). Absorption rates are given as percentages of optical densities obtained with and without absorption. Circles represent the mean absorption rates of five wells, error bars the standard errors. Antiganglioside lgG antibodies did not cross react with $H$ influenzae LOS in patients with serological evidence of recent $C$ jejuni infection, whereas the antibodies did react with the isolate LOS in those with serological evidence of recent $H$ influenzae infection. $A b$, antibody. has been isolated from some GBS/Fisher syndrome patients who experienced only upper respiratory infectious symptoms or fever. ${ }^{10}$ Our findings suggest that $H$ influenzae isolation does not always indicate that this is the causative agent in GBS and Fisher syndrome. Even when $H$ influenzae is isolated, we should also test for other infections-in particular $C$ jejuni-irrespective of the antecedent symptoms. This also may hold true for cases in which other agents are isolated and serological evidence obtained. We believe that more careful judgment of the causal agent in GBS and Fisher syndrome will lead to a decrease in the numerous case reports of sham causal infection in these syndromes.

Positive $H$ influenzae serology was found in $26 \%$ of the tested cases in whom $H$ influenzae had been isolated. This low frequency may partly reflect the assay's low sensitivity, or it may indicate that $H$ influenzae infection was merely a complication of GBS or Fisher syndrome in some of the patients, because most of the serum samples assayed were obtained soon after neurological onset, at a time when neither antibody response nor $H$ influenzae infection may have occurred. However, anti-GQlb and anti-GTla antibodies were detected in most of the $H$ influenzae seropositive patients, all of whom had Fisher syndrome. This supports our previous results showing that $H$ influenzae infection is associated more closely with Fisher syndrome than with GBS and that production of antiganglioside antibody is mediated by a GQlb/GTla epitope on the bacterial LOS. ${ }^{4}$ A serological $H$ influenzae test and examination of the cross reactivity of antiganglioside antibodies with the isolate LOS would help in determining the causal role of the bacterium when $H$ influenzae is isolated in such cases.

\section{ACKNOWLEDGEMENTS}

We thank Ms Maki Okazaki for her excellent support in all the assays. Supported in part by grants from the Ichiro Kanehara Foundation to MK; the Kanae Foundation for Life \& Socio-Medical Science to MK; the Japan Intractable Diseases Research Foundation to MK; a grant for Scientific Research (B) (KAKENHI 14370210 to NY) from the Ministry of Education, Culture, Sports, Science and Technology of Japan; a Research Grant for Neuroimmunological Diseases to NY from the Ministry of Health, Labour and Welfare of Japan; a Health Sciences Research Grant (Research on Psychiatric and Neurological Diseases and Mental Health) to NY from the Ministry of Health, Labour, and Welfare of Japan; and a grant from the Human Frontier Science Program (RGP0038/2003-C to NY).

\section{Authors' affiliations}

M Koga, K Hirata, N Yuki, Department of Neurology, Dokkyo University School of Medicine, Tochigi, Japan

S Koike, Institute for Medical Science, Dokkyo University School of Medicine

Competing interests: none declared.

Correspondence to: Dr Michiaki Koga, Department of Neurology, Dokkyo University School of Medicine, Kitakobayashi 880, Mibu, Shimotsuga, Tochigi 321-0293, Japan; kogamrk@dokkyomed.ac.jp

Received 10 February 2005

In revised form 24 March 2005

Accepted 30 March 2005 


\section{REFERENCES}

1 Jacobs BC, Rothbarth PH, van der Meche FGA, et al. The spectrum of antecedent infections in Guillain-Barre syndrome. A case-control study. Neurology 1998;51:1110-15.

2 Ropper AH, Wijdicks EFM, Truax BT, eds. Guillain-Barre syndrome. Philadelphia: FA Davis Co, 1991.

3 Mori M, Kuwabara S, Miyake M, et al. Haemophilus influenzae infection and Guillain-Barre syndrome. Brain 2000;123:2171-8.

4 Koga M, Yuki N, Tai T, et al. Miller Fisher syndrome and Haemophilus influenzae infection. Neurology 2001;57:686-91.

5 Ju YY, Womersley H, Pritchard J, et al. Haemophilus influenzae as a possible cause of Guillain-Barre syndrome. J Neuroimmunol 2004;149:160-6.
6 Koga M, Gilbert M, Li J, et al. Antecedent infections in Fisher syndrome: a common pathogenesis of molecular mimicry. Neurology 2005;64:1605-11.

7 Kuwabara S, Mori M, Ogawara K, et al. Indicators of rapid clinical recovery in Guillain-Barre syndrome. I Neurol Neurosurg Psychiatry $2001 ; 70: 560-2$

8 Yuki N, Tagawa Y, Irie F, et al. Close association of Guillain-Barre syndrome with antibodies to minor monosialogangliosides GMIb and GM1 $\alpha$. J Neuroimmunol 1997;74:30-4.

9 Willison HJ, Yuki N. Peripheral neuropathies and anti-glycolipid antibodies. Brain 2002;125:2591-625.

10 Takahashi M, Koga M, Yokoyama K, et al. Epidemiology of Campylobacter jejuni isolated from patients with Guillain-Barre and Fisher syndromes in Japan. J Clin Microbiol 2005;43:335-49.

\section{NEUROLOGICAL PICTURE}

\section{Ultra fast resolution of acute post-traumatic subdural haematoma}

$\mathrm{P}$ ost-traumatic acute subdural haematoma (ASDH) is a life threatening condition. The traditional approach is urgent surgery in haematomas with a mass effect. We report a case with a large post-traumatic ASDH whose haematoma disappeared markedly within 2 hours.

A 19 year old man sustained a head trauma at 22:30 and presented with Glasgow Coma Scale (GCS) score of 9 to the state hospital. CT scan showed a right hemispheric ASDH and a marked midline shift (panel A). He was transferred to our hospital for urgent surgery. In our hospital his GCS score was 12. A repeat CT scan at 00:30 showed substantial decrease of ASDH and oedema (panel B). Six hours later CT revealed total resolution of ASDH and the appearance of subarachnoidal haemorrhage (panel $\mathrm{C}$ ). On the third day he was completely alert with a GCS of 15 . MRI on the fifth day showed mild subarachnoidal haemorrhage (panel D). He was discharged on the tenth day with excellent neurological condition.

Although infrequently reported, rapid spontaneous resolution of an ASDH may be underestimated. ${ }^{1-5}$ Two possible hypotheses for spontaneous resolution have been proposed: (1) the haematoma is diluted by cerebrospinal fluid and is washed out; (2) the haematoma is compressed by the pressure produced by acute cerebral swelling and redistributed. ${ }^{2}$ Some authors have demonstrated redistribution and dispersal of blood in the subdural space with MRI. ${ }^{45}$

We think that a tear in the arachnoid membrane and connection between the subdural and subarachnoidal spaces results in dilution of haematoma. Thus not only redistribution and dispersal but also washing out of haematoma by retrograde flow into the subarachnoidal space may be the causes of ultra fast spontaneous resolution of an ASDH. In cases showing rapid improvement of neurological status a subsequent control CT is indicated before surgery.

H Mirzai
C Yaldiz
M Eminoglu
Department of Neurosurgery, Celal Bayar University, School of Medicine,
Manisa, Turkey
S Orguc
Department of Radiology, Celal Bayar University, School of Medicine,
Manisa, Turkey

Correspondence to: Dr Hasan Mirzai, 2040 Sok. Pamukkale 4/60 D:67, Mavisehir, Atakent, 35540, Izmir Turkey; hmirzai@yahoo.com

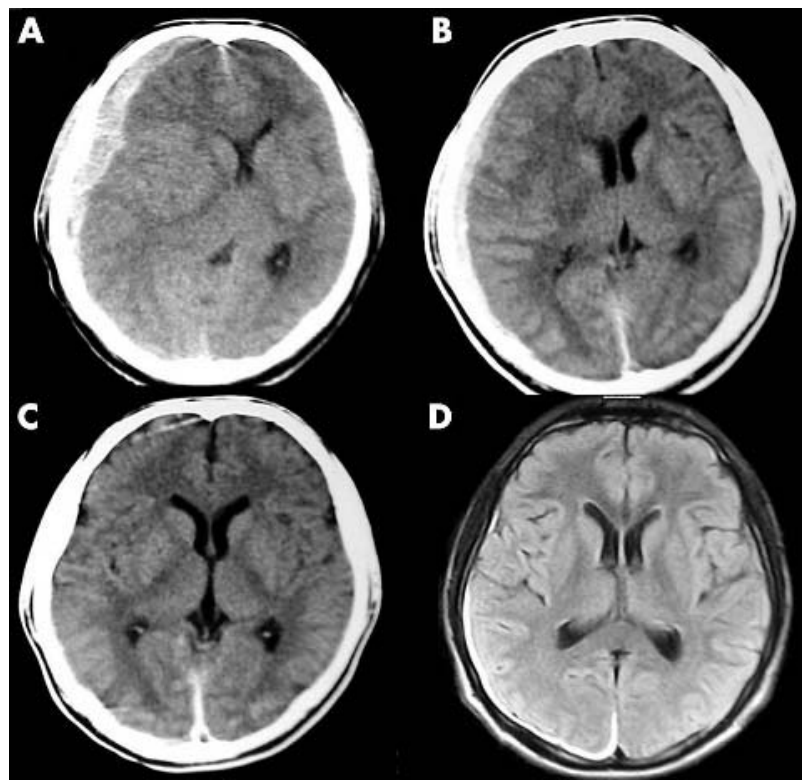

Figure 1 (A) Initial CT scan shows subdural haematoma in the right frontoparietal region and a marked midline shift; (B) Post-traumatic second hour CT shows substantial decrease in oedema and size of subdural haematoma; (C) Post-traumatic eighth hour CT shows total resolution of the subdural haematoma and the appearance of subarachnoidal haemorrhage; (D) Fifth day MRI shows mild subarachnoidal haemorrhage.

\section{References}

1 Fernandez-Portales I, Gomez-Perals L, Cabezudo JM, et al. Rapid spontaneous resolution of acute subdural hematoma. Neurocirugia (Astur) 2002;3:491-4

2 Kuroiwa T, Tanabe H, Takatsuka $\mathrm{H}$, et al. Rapid spontaneous resolution of acute extradural and subdural hematomas. Case report. J Neurosurg 1993;78:126-8.

3 Berker M, Gulsen S, Ozcan OE. Ultra-rapid spontaneous resolution of acute posttraumatic subdural hematomas in patient with temporal linear fracture. Acta Neurochir 2003;145:715-7.

4 Polman CH, Gijsbers CJ, Heimans JJ, et al. Rapid spontaneous resolution of an acute subdural hematoma. Neurosurgery 1986;19:446-8.

5 Kato N, Tsunoda T, Matsumura A, et al. Rapid spontaneous resolution of acute subdural hematoma occurs by redistribution - two case reports. Neurol Med Chir (Tokyo) 2001;41:140-3. 